\title{
Temporal dynamics of focal point location under self-focusing of nanosecond laser pulses
}

\author{
${ }^{1}$ Ivanisik A., ${ }^{1}$ Korotkov P. and ${ }^{2}$ Ponezha G. \\ ${ }^{1}$ Kyiv National Taras Shevchenko University, 4-g Glushkova Avenue, 03022 Kyiv, \\ Ukraine, e-mail: aivan@univ.kiev.ua \\ ${ }^{2}$ National Academy of Statistics, Accounting and Auditing, 1 Pidgirna Street, 04107 \\ Kyiv, Ukraine
}

Received: 21.09 .2013

\begin{abstract}
Temporal dynamics of location of a focal point due to self-focusing of giant nanosecond laser pulses is analyzed. The location, velocity and the acceleration of the focal point are determined with taking the group speed of laser radiation into consideration. It is shown that the focal point velocity can vary from $-\infty$ to $+\infty$ but, at the output of a long Kerr cell, it is close to the group speed of light. At the front of the pulse, this velocity slightly exceeds the group speed of the pulse and becomes less than the group speed at the rear edge of the pulse. Under minimal self-focusing distance at the top of the laser pulse, the focal point velocity becomes zero. The acceleration of the focal point at the exit of a long cell can be almost zero, though it acquires a positive value at the minimal focal distance. Our results are essential while determining the efficiency of parametric stimulated Raman scattering, which requires both phase and group synchronisms along the laser pulse trace through a typical Kerr fluid.
\end{abstract}

Keywords: Kerr effect, self-focusing, stimulated Raman scattering

PACS: 42.65.Dr

UDC: 535.375

\section{Introduction}

In optical media of limited lengths, high powers of light induce nonlinear optical processes such as a stimulated Raman scattering. These processes are mainly observed using pulsed lasers, with laser pulse durations changing from tens of nanoseconds to tens of femtoseconds, or even less. The duration of pulses affects significantly dynamics of the nonlinear processes, because the position of a region of induced nonlinear polarization and the amplitude of this polarization are changed while the pulse is developing.

The problem of influence of nanosecond-long pulse envelopes in a Kerr medium is very difficult [1-4]. As a consequence of self-focusing, changes in the instantaneous power of a pulse cause changing cross-section of a laser ray and displacing region of a maximal light intensity. The efficiency of the nonlinear optical processes increases in the close vicinity of a self-focusing point, where the intensity is maximal (see, e.g., Ref. [5] regarding the relevant results for the stimulated Raman scattering). So, the spatial region where the nonlinear optical processes take place moves, too. Though such a situation is close to the case of more short pulses of picosecond or femtosecond durations, it has some additional peculiarities $[6,7]$.

In the case of picosecond or femtosecond pulses, the region of the maximum nonlinear polarization amplitude moves almost with the group speed of light, while in the case of nanosecond pulses it moves with the velocity of a focal point of self-focusing. Depending on laser pulse dura- 
tion, the focal point velocity changes from zero near the pulse top to superluminal on the pulse front. Moreover, the sign of the velocity changes from positive, when the focal region moves in the direction of light ray, to negative in the opposite case. Thus, simultaneous consideration of the group and phase synchronisms becomes a difficult problem whenever the velocity of the focal point of self-focusing replaces the group speed of light.

The situation when the velocity of the region with the maximal nonlinear polarization amplitude is close to zero is equivalent to that occurring when conventional lenses are used, for any durations of laser pulses. However, for high enough velocities of the focal point and especially for superluminal ones, a specific situation takes place which is impossible for achieving under different conditions or by other facilities. At the superluminal speeds, there arise certain analogies with Cherenkov's radiation $[8,9]$. Thus, the nonlinear optical processes excited by laser pulses of nanosecond durations extend a range of possible implementations of the nonlinear optical processes, and the same refers to the linear optical processes, too.

We emphasize that independence of the nonlinear polarization region velocity of the group and phase light speeds is in general a unique case and, perhaps, it is peculiar only to the nanosecond range of laser pulses. In this respect, theoretical and experimental studies of parametric nonlinear optical processes, which require the conditions of the group and phase synchronisms, are very important under self-focusing. In the situation of variable velocity and acceleration of the region where nonlinear polarization exists under self-focusing, nonlinear optical processes become dynamic, thus affecting the resulting spectrum of the scattered light.

Earlier, studies with this formulation of the question have not been systematic, in spite of their topical character. It has been generally accepted that the region of nonlinear polarization is limited in space and does not move under focusing by conventional lenses, or approaches the group speed of exciting pulses. Our present research has been motivated by the fact that modern technologies have progressed so drastically that, in principle, the group velocity of light can vary [9] and the shape of pulses can be controlled. The purpose of this article is to ascertain the location, the velocity and the acceleration of the focal point appearing under self-focusing in Kerr liquids for the case of nanosecond laser pulses. These characteristics have a significant effect on the dynamics and efficiency of the nonlinear optical phenomena occurring along the pulse trace.

\section{General relations for the velocity of focal point under self-focusing}

Theoretical description of self-focusing is based on the Maxwell equations and is usually carried out for a stationary $(s t)$ dependence of a focal point $(f p)$ location $z_{f p}^{(s t)}=z_{f p}\left\{P_{L}=\right.$ const $\}$, where $z$ is a longitudinal coordinate and $P_{L}$ a laser power. However, the value $z_{f p}^{(s t)}$ describes a focal distance $z_{f d}$ at the laser power $P_{L}=$ const, with no account of the laser radiation group speed $v_{g L}$ under the condition $P_{L} \neq$ const. Experimenters must take into consideration such a peculiarity of description of the self-focusing effect. In particular, it is necessary to distinguish between the focal point location $\left(z_{f p}\right)$ and the focal distance $\left(z_{f d}\right)$ itself.

If the laser power at the input of a medium $(z=0)$ changes with time $t$, then we have $z_{f p}\left\{P_{L}\{t, z=0\}, v_{g L}=\infty\right\}=z_{f p}^{(s t)}\{t\}=z_{f d}\{t\}$ in the approximation given by $v_{g L}=\infty$. The value $z_{f d}\{t\}$ is the focal distance of the self-focusing for each of laser pulse fragments. However, it takes some time for each of these fragments to reach the focal point $z_{f p}$. 
The temporal dependence of the focal point velocity $v_{f p}=\partial z_{f p} / \partial t$ can be analyzed analytically with the known quasi-stationary relationship $z_{f d}\{t\}$, which does not take into account a delay $z / v_{g L} \neq 0$. Formally, we have

$$
\begin{aligned}
v_{f p} & =\partial z_{f p} / \partial t= \\
& =\left(\partial z_{f p} / \partial\left(t-z_{f p} / v_{g L}\right)\right)\left(\partial\left(t-z_{f p} / v_{g L}\right) / \partial t\right)= \\
& =\left(\partial z_{f p}\left\{P_{L}\left\{t-z_{f p} / v_{g L}\right\}\right\} / \partial\left(t-z_{f p} / v_{g L}\right)\right)\left(1-v_{f p} / v_{g L}\right) .
\end{aligned}
$$

Here the following question appears: what is meant by the expression $\partial z_{f p}\left\{P_{L}\left\{t-z_{f p} / v_{g L}\right\}\right\} / \partial\left(t-z_{f p} / v_{g L}\right)$ obtained with replacing $t \rightarrow\left(t-z_{f p} / v_{g L}\right)$ ? Changing the variable $t$ by $\left(t-z_{f p} / v_{g L}\right)$ gives a derivative with the time correction in the interval of moving to the point $z=z_{f p}$. In other words, the value $\partial z_{f p}\left\{t-z_{f p} / v_{g L}\right\} / \partial\left(t-z_{f p} / v_{g L}\right)$ represents a velocity of the focal distance for an instantaneous pulse power at the entrance boundary of a nonlinear optical medium, which equals to $P_{L}\left\{t-z_{f p} / v_{g L}\right\}$. Therefore one can write

$$
\frac{\partial z_{f p}\left\{P_{L}\left\{t-z_{f p} / v_{g L}\right\}\right\}}{\partial\left(t-z_{f p} / v_{g L}\right)}=v_{f d} .
$$

With the notation $v_{f d}$ for the velocity of the focal distance, the following analytical dependence may be obtained from Eqs. (1) and (2):

$$
\frac{1}{v_{f p}}=\frac{1}{v_{f d}}+\frac{1}{v_{g L}} .
$$

Eq. (3) relates the group speed $v_{g L}$ of laser radiation, the focal point velocity $v_{f p}\{t, z\}$, and the focal distance velocity $v_{f d}\left\{t-z_{f p} / v_{g L}, z\right\}$ at the moment $t-z_{f p} / v_{g L}$, when a self-focusing fragment of the laser pulse is at the entrance boundary.

The expression given by Eq. (3) may be derived in somewhat different manner understood more easily from the physical point of view. Namely, let us analyze the velocities $v_{f p}$ and $v_{f d}$ near the coordinate $z$. The focal distance $z_{f d}$ is equal to $z$ at the moment $t_{f d}\{z\}$, and we have $z_{f p}=z$ at the moment

$$
t_{f p}\{z\}=t_{f d}\{z\}+\frac{z}{v_{g L}},
$$

with the delay $z / v_{g L}$ with respect to $t_{f d}\{z\}$.

Further, we are to consider a shift in the coordinate $z$ by an infinitesimal value $d z$. For this coordinate $z+d z$, the equality $z_{f d}=z+d z$ is fulfilled at the time moment $t_{f d}\{z+d z\}=t_{f d}\{z\}+d z / v_{f d}$, and the equality $z_{f p}=z+d z$ is valid for the moment

$$
t_{f p}\{z+d z\}=t_{f d}\{z+d z\}+\frac{(z+d z)}{v_{g L}}=t_{f d}\{z\}+\frac{d z}{v_{f d}}+\frac{(z+d z)}{v_{g L}} .
$$


Issuing from the physical meaning of the velocity of the focal point, we obtain

$$
v_{f p}=\frac{d z}{t_{f p}\{z+d z\}-t_{f p}\{z\}} .
$$

After substituting Eqs. (4) and (5) into Eq. (6), we arrive at the relation

$$
v_{f p}=\frac{d z}{d z / v_{f d}+d z / v_{g L}} .
$$

Reduction of the variable $d z$ in Eq. (7) yields in Eq. (3). Then Eq. (3) may be represented as

$$
v_{f p}=\frac{v_{f d} v_{g L}}{\left(v_{f d}+v_{g L}\right)} .
$$

Analysis of Eqs. (3) or (8) shows that, under approximation $v_{g L}=\infty$, the equality $v_{f p}=v_{f d}$ takes place, i.e. the velocity of the focal point coincides with the focal distance velocity. Certainly, it had to be expected with the definitions of $v_{f p}$ and $v_{f d}$ introduced earlier. On the other hand, for rapid enough $z_{f d}$ changes (i.e., at the front and the rear of the laser pulse), the velocity $v_{f d}$ can acquire the values $v_{f d}=\rightarrow \mp \infty$, respectively. So, we get $v_{f p} \rightarrow v_{g L}$, regardless of the sign of the velocity $v_{f d}$. In general, the equality $v_{f p} \approx v_{g L}$ is typical for the ultrashort pulses beginning from the picosecond range, though it is far from being so for the nanosecond pulses.

At the front of nanosecond pulses in long media, the value $v_{f d}$ changes from $v_{f d} \rightarrow-\infty$ to zero respectively at the 'wing' and the top of the pulse. The velocity $v_{f p}$ of the focal point acquires the values from $\left.v_{f p}\right|_{v_{f d} \rightarrow-\infty} \approx v_{g L}$ to $\left.v_{f p}\right|_{v_{f d}} \approx-v_{g L} \rightarrow+\infty$. After $v_{f d}=-v_{g L}$, the velocity $v_{f p}$ changes its sign and varies from $\left.v_{f p}\right|_{v_{f d} \approx-v_{g L}} \rightarrow-\infty$ to $\left.v_{f p}\right|_{v_{f d}=0}=0$ afterwards. At the rear edge of the laser pulse, the velocity of the focal distance $v_{f d}$ increases from zero to $+\infty$. This leads to increasing focal point velocity $v_{f p}$ from $\left.v_{f p}\right|_{v_{f d}=0}=0$ to $\left.v_{f p}\right|_{v_{f d} \rightarrow \infty} \approx v_{g L}$. A more detailed description of the behaviour of $v_{f p}\left\{v_{f d}\right\}$ is illustrated in Fig. 1. It is seen that the focal point velocity changes in a wide range for the rising and falling edges of nanosecond laser pulses. Obviously, such a situation represents a dynamic effect which imposes additional features on the evolution of optical processes in self-focusing media.

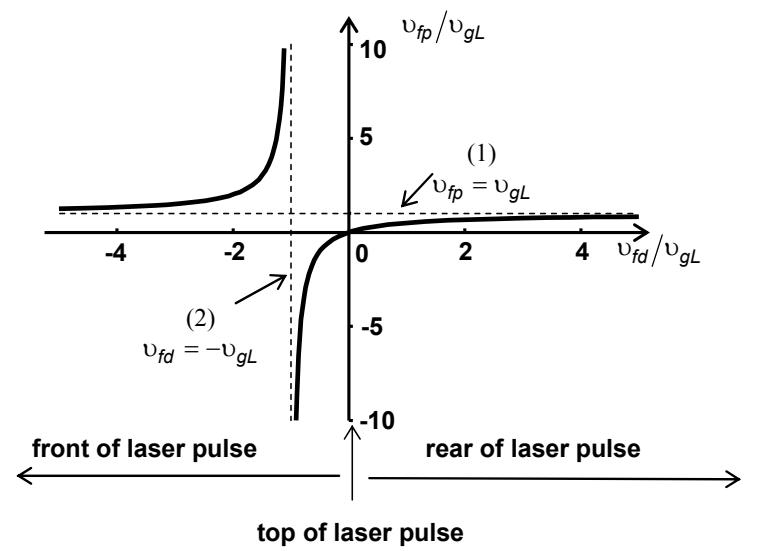

Fig. 1. Dependence of focal point velocity $v_{f p}$ on focal distance velocity $v f d$. Both velocities are normalized to the group speed $v_{g L}$ of laser radiation. The velocity $v f d$ is negative at the front of a laser pulse, zero at its top, and positive at the rear edge. The asymptotes are (1) $\cup_{f p} \rightarrow \cup_{g L}$ at $\cup_{f d} \rightarrow \mp \infty$ and (2) $v_{f p} \rightarrow \pm \infty$ in the vicinity of $v_{f d}=-v_{g L}$. 


\section{Location, velocity and acceleration of the focal point}

Quantitative description of the velocity or the acceleration of the focal point due to the selffocusing should involve, at the least, specifying dependences of the stationary focal distance on the light power at the input of the nonlinear optical medium.

For the input laser power $P_{L}\{t, z=0\}$ greater than the critical self-focusing power $P_{c r}$, the dependence of the focal distance $z_{f d}$ on $\tilde{P}=P_{L} / P_{c r}$ is described by the expression confirmed experimentally for a passively modulated ruby laser at the wavelength $\lambda=0.69 \mu \mathrm{m}$ under smallscale self-focusing [11],

$$
z_{f d}=\frac{0.38 k_{L} a_{0}^{2}}{\sqrt{(\sqrt{\tilde{P}}-0.77)^{2}-0.053}} .
$$

In Eq. (9) $k_{L}$ denotes the module of the wave vector of laser radiation in the medium, $a_{0}$ the halfwidth of the transversal intensity distribution at the level $e^{-1}$ at the entrance boundary of that medium (notice that this distribution is assumed to be Gaussian). Under small-scale self-focusing, $a_{0}$ acquires the value $\sim 100 \mu \mathrm{m}$ [12]. Note that Eq. (9) is similar to the Marburger equation [13].

The dependences $z_{f d}\{t\}, z_{f p}\{t\}$ and $v_{g L} t$ typical for the laser pulse passing through a toluene cell are given in Fig. 2. Here we have assumed that the pulse (or the subpulse) has a Gaussian power profile with a constant time $\tau=1 \mathrm{~ns}$. We have made use of the following parameters: $\tilde{P}=P_{L}\{t=0, z=0\} / P_{c r} \equiv \tilde{P}_{0}=3.6, a_{0}=113 \mu \mathrm{m}, v_{g L}=0.65662 c$ (with $c$ being the absolute light speed in vacuum), and $k_{L}=134887 \mathrm{~cm}^{-1}$. The time $t=0$ is associated with the top of the laser pulse at $z=0$.

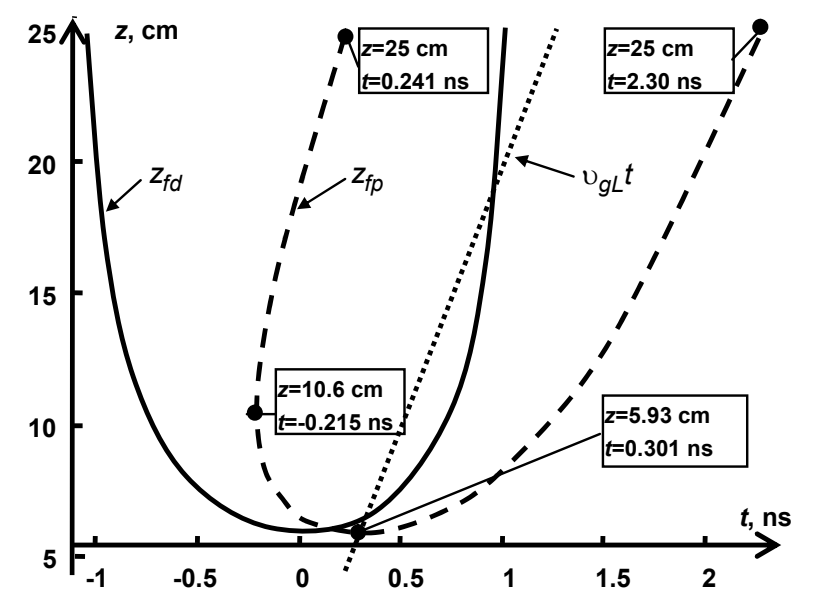

Fig. 2. Locations of focal distance $z_{f d}$ (solid curve), focal point $z_{f p}$ (dash curve) and a point $z=v_{g L}$ (dot curve) in the coordinates $z\{t\}$ for a laser pulse with the parameters $\lambda=0.69 \mu \mathrm{m}, \quad \tau=1 \mathrm{~ns}, \quad \tilde{P}_{0}=3.6 \quad$ and $a_{0}=113 \mu \mathrm{m}$ propagating in toluene. Characteristic points for $z_{f p}$ are andicated by dots of bold type

The values $z_{f d}\{t\}$ and $z_{f p}\{t\}$ are linked by the equation $z_{f p}\{t\}=z_{f d}\left\{t+z_{f d} / v_{g L}\right\}$, which has no analytical form and so has been solved numerically. The difference of $z_{f p}\{t\}$ from $z_{f d}\{t\}$ increases with deviation of $t$ from a zero located at the top of the pulse. While approaching $v_{g L}=\infty$, the both dependences coincide and $v_{g L} t$ becomes a vertical line at $t=0$. 
Under the same conditions as in Fig. 2, we have calculated numerically the inverse $z_{f p}\{t\}$ function, i.e. the function $t\left\{z=z_{f p}\right\}$. The results are shown in Fig. 3. Here one can take notice of a moment of time when the focal point has a certain coordinate $z$ (e.g., at the entrance boundary of a cell with the length $L=z=z_{f p}$ ). This dependence is important for understanding the results concerned with the velocity and the acceleration of the focal point of self-focusing at a fixed distance.

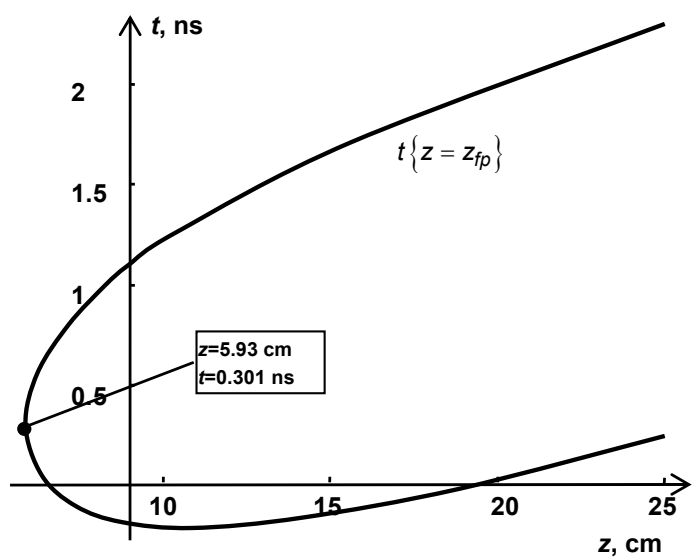

Fig. 3. Locations of focal point $z f p$ in the coordinates $t\{z\}$ for a laser pulse with the parameters $\lambda=0.69 \mu \mathrm{m}, \quad \tau=1 \mathrm{~ns}, \quad \tilde{P}_{0}=3.6$ and $a_{0}=113 \mathrm{mkm}$ propagating in toluene.

Fig. 4 presents dependence of the normalized focal point velocity $v_{f p} / v_{g L}$ on the time $t$, with the limit $L=z \leq 25 \mathrm{~cm}$ used. We are to point out the following features: there is a singularity $v_{f p}= \pm \infty$ at the pulse front (i.e., at $t=0.215 \mathrm{~ns}$ - see Fig. 2), whereas at the both pulse 'wings' the velocity of the focal point asymptotically approaches $v_{g L}$.

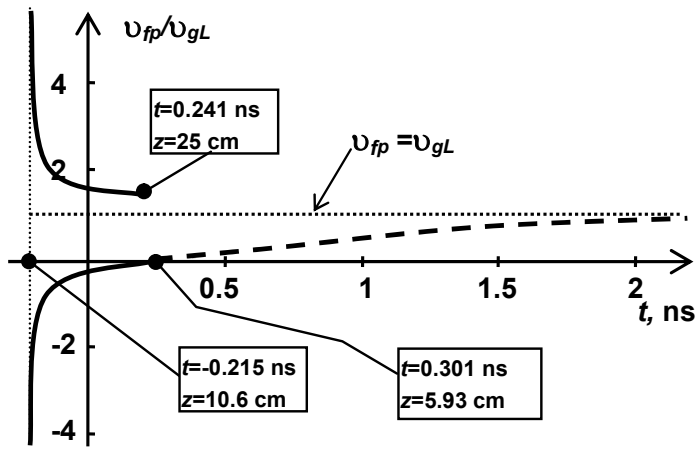

Fig. 4. Normalized focal point velocity $v_{f p} / v_{g L}$ in the coordinates $v f p\{t\}$ for a laser pulse with the parameters $\lambda=0.69 \mu \mathrm{m}, \quad \tau=1 \mathrm{~ns}, \quad \tilde{P}_{0}=3.6$ and $a_{0}=113 \mu \mathrm{m}$ propagating in $L=25 \mathrm{~cm}$ long toluene cell. Solid curve corresponds to the front edge and dash curve to the rear edge of the pulse.

The parameters of the scattered radiation can be determined experimentally [5] along the path of a laser pulse through a cell and at its exit. That is why it is important to clarify the dependence $v_{f p}\{z\}$. The appropriate results of calculations are displayed in Fig. 5. For better understanding of the dependence, one should make use of Fig. 3 and take into account that $z_{f p}=z$.

The velocity of the focal point influences the group synchronism. Then the acceleration of the focal point is also important, since it determines the distance where the group synchronism is achieved. We emphasize here that the group and phase synchronisms in self-focusing media are closely linked with each other. Moreover, the acceleration of the focal point may be examined as a function of time (see Fig. 6). 

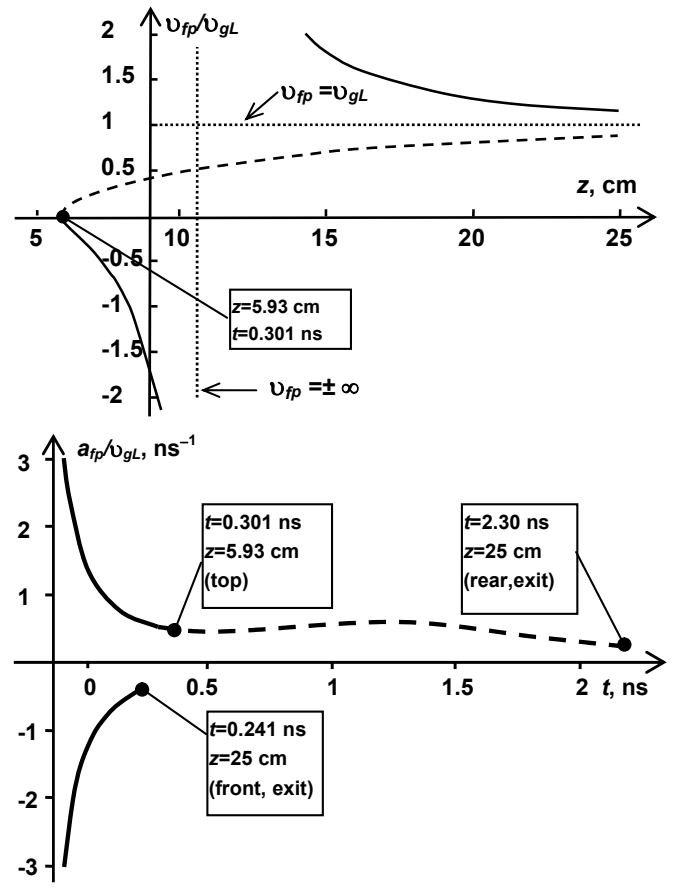

Fig. 5. Normalized focal point velocity $v_{f p} / v_{g L}$ in the coordinates $v_{f p}\{z\}$ for a laser pulse with the parameters $\lambda=0.69 \mu \mathrm{m}, \quad \tau=1 \mathrm{~ns}, \quad \tilde{P}_{0}=3.6 \quad$ and $a_{0}=113 \mu \mathrm{m}$ propagating in toluene. Solid curve corresponds to the front edge and dash curve to the rear edge of the pulse.

Fig. 6. Focal point acceleration $a_{f p}\{t\}$ for a laser pulse with the parameters $\lambda=0.69 \mu \mathrm{m}, \tau=1 \mathrm{~ns}$, $\tilde{P}_{0}=3.6$ and $a_{0}=113 \mathrm{mkm}$ propagating in $L=25 \mathrm{~cm}$ long toluene cell. Solid curve corresponds to the front edge and dash to the rear edge of the pulse.

For understanding experimental dependences of the efficiency of stimulated Raman scattering on the longitudinal coordinates, one should know the coordinate dependence of the focal point acceleration rather than the temporal one. This dependence is shown in Fig. 7, with the laser pulse parameters taken the same as above.

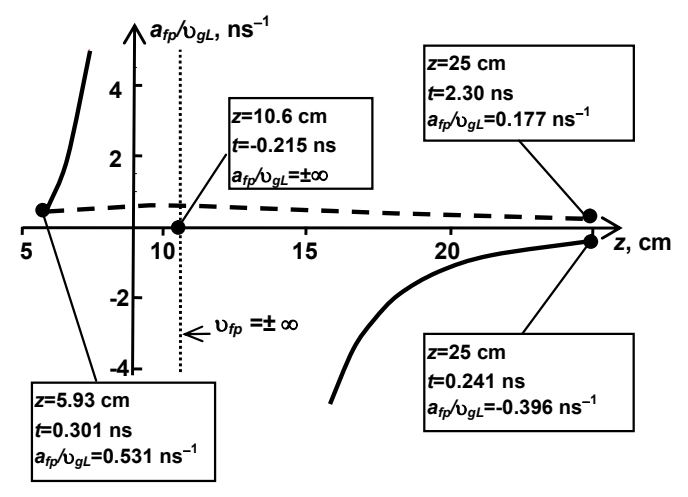

Fig. 7. Normalized acceleration $a_{f p} / \mathrm{v}_{g L}$ of focal point in the coordinates $a_{f p}\{z\}$ for a laser pulse with the parameters $\lambda=0.69 \mu \mathrm{m}, \tau=1 \mathrm{~ns}, \tilde{P}_{0}=3.6$ and $a_{0}=113 \mu \mathrm{m}$ propagating in $L=25 \mathrm{~cm}$ long toluene cell. Solid curve corresponds to the front and dash curve to the drop of the pulse.

\section{Conclusion}

We have analyzed the effect of self-focusing in a Kerr liquid in the nanosecond range of laser pulse durations. The results concern the location, velocity and the acceleration of a focal point appearing due to self-focusing effect. The influence of the group speed of laser pulses is taken into consideration.

We have shown that the focal point velocity can acquire arbitrary values from $-\infty$ to $+\infty$. However, the focal point velocity is close to the group speed of light at the output of a long cell filled with the Kerr liquid. At the front of the pulse, this velocity becomes somewhat higher than the group speed of the pulse, though it remains slightly less than the group speed at the rear edge of the latter. Under minimal self-focusing distance at the top of the laser pulse, the focal point velocity is zero. The acceleration of the focal point can be almost zero at the exit of a long cell, but it 
has a fixed positive value at the minimal focal distance.

The results obtained here make possible predicting efficiency of the parametric stimulated Raman scattering along the laser pulse trace through a Kerr medium. In particular, the efficiency of the stimulated Raman scattering can increase at the top of the pulse where the speed of the focal point is zero. In addition, this efficiency can become significant at the front edge of the pulse just exiting a long cell, under which conditions the group synchronism can be achieved.

\section{References}

1. Loy M and Shen Y, 1973. Study of self-focusing and small-scale filaments of light in nonlinear media. IEEE J. Quant. Electron. 9: 409-422.

2. Shen Y. The principles of nonlinear optics, Hoboken, New Jersey: Wiley Classics Library (2002).

3. Boyd R, Lukishova S and Shen Y, Self-focusing: past and present. Berlin: Springer (2009)

4. Chekalin S V and Kandidov V P, 2013. From self-focusing light beams to femtosecond laser pulse filamentation. Phys.-Usp. 56: 123.

5. Ivanisik A, Malyi V and Ponezha G, 1996. Spatial-angular structure of anti-Stokes radiation formed by stimulated Raman scattering in a Kerr liquid. Opt. Spectr. 80: 185-189.

6. Ivanisik A I, and Ponezha G V, 2001. Spectrum of anti-Stokes stimulated Raman scattering from the moving focal regions of self-focusing. Opt. Spectr. 90: 625-629.

7. Dudka S, Ivanisik A, Konopatskiy A and Korotkov P, 2006. Transition effect at the mediumvacuum interface under the self-phase modulation of a light pulse. Ukr. J. Phys. 51: 140-146.

8. Ivanisik A, Malyi V and Ponezha G, 1997. Cherenkov-type radiation under conditions of Raman light scattering in self-focusing liquids. Opt. Spectr. 82: 410-415.

9. Ivanisik A, Isaienko O, Korotkov P and Ponezha G, 2012. Phase-modulated parametric antiStokes stimulated Raman scattering of Cherenkov-type in self-focusing areas of exciting radiation. Ukr. J. Phys. 57: 1000-1010.

10. Shcherbin K, Mathey P, Gadret G, Guyard R, Jauslin H and Odoulov S, 2013. Slowing down of light pulses using photorefractive four-wave mixing: Nontrivial behavior with increasing coupling strength. Phys. Rev. A. 87: 033820.

11. Dudka S, Ivanisik A, Konopatskiy A and Loboda V, 2005. Disposition of the focal point in a self-focusing environment. Bulletin of T. Shevchenko Nat. Univ. Kyiv, Ser. Phys. \& Math. 3: 381-388.

12. Ivanisik A, Malyi V and Ponezha G, 1998. Effect of self-focusing on the angular spectra of stimulated Raman scattering. Opt. Spectr. 85: 78-84.

13. Marburger J, 1975. Self-focusing: theory. Prog. Quantum Electron. 4: 35-110.

Ivanisik A., Korotkov P. and Ponezha G. 2014. Temporal dynamics of focal point location under self-focusing of nanosecond laser pulses. Ukr.J.Phys.Opt. 15: $1-8$.

Анотація. Аналізується ефект самофокусування в керрівських рідинах для наносекундних лазерних імпульсів. Подано результати, що стосуються розташування, швидкості та прискорення фокальної точки самофокусування. Враховано вплив групової швидкості лазерних імпульсів. Швидкість фокальної точки змінюсться в межах $\pm \infty$, проте на виході протяжної кювети 3 керрівською рідиною ия швидкість близька до групової швидкості світла. На фронті імпульсу швидкість фокальної точки дещо перевищує групову швидкість імпульсу та менша на спаді. $3 a$ мінімальної відстані самофокусування на вершині імпульсу швидкість фокальної точки нульова. Прискорення фокальної точки на виході довгих кювет може бути практично відсутнім, але за мінімальної фокальної відстані набуває фіксованого додатного значення. Наведені результати дають змогу прогнозувати ефективність вимушеного параметричного комбіначійного розсіювання за трасою проходження лазерного випромінювання крізь типове керрівське середовище. 